\title{
Angioplastia "Primária" de Artéria Carótida Interna Direita em Paciente com Contraindicação à Terapia Trombolítica
}

\author{
Guilherme Ferragut Attizzani', Yourki Tairouz', Ramón Cedeño Ecarri'
}

\begin{abstract}
RESUMO
É relatado o caso de paciente do sexo masculino, de 63 anos de idade, que apresentava história prévia de acidente vascular encefálico isquêmico, com sequela motora e oclusão da artéria carótida interna esquerda. O paciente foi admitido no serviço de emergência com episódio agudo (quatro horas após o início dos sintomas) de novo evento isquêmico cerebrovascular. Apresentava contraindicação absoluta para ser submetido a terapia trombolítica, em decorrência da recente realização de biópsia hepática. Assim, optou-se pela realização, na fase aguda, de angiografia de artérias carótidas e vertebrais. A lesão culpada foi identificada e procedeu-se ao implante de stent autoexpansível, obtendo-se sucesso angiográfico e melhora clínica substancial. Embora não exista substrato robusto na literatura que suporte a conduta adotada (angioplastia "primária" da artéria carótida interna), essa pareceu ser a melhor estratégia a ser implementada naquele momento.
\end{abstract}

DESCRITORES: Artérias carótidas. Doenças das artérias carótidas. Acidente cerebral vascular. Angioplastia.

E tima-se que $85 \%$ dos acidentes vasculares encefálicos sejam de origem isquêmica ${ }^{1}$. A fase aguda dessa entidade patológica é ameaçadora à vida, e, particularmente, a oclusão da artéria carótida interna pode levar à morte em cerca de $20 \%$ dos casos e a sequelas graves permanentes em torno de $40 \%$ dos pacientes ${ }^{2}$, além de exibir altos índices de recorrência ${ }^{3,4}$. A administração de terapia trombolítica (rt-PA) por via intravenosa tem sua indicação precisa dentro de três horas a partir do início dos sintomas, levando à redução significativa da incapacidade funcional ${ }^{5}$. Entretanto, há algumas contraindicações abso-

1 Hospital Metropolitano del Norte - Valencia, Venezuela. Correspondência: Guilherme Ferragut Attizzani. Av. Pedro Soares de Camargo, 198/51 - Anhangabaú - Jundiaí, SP, Brasil - CEP 13208-080 E-mail: gfattizzani@hotmail.com

Recebido em: 2/7/2009 • Aceito em: 15/10/2009

\section{ABSTRACT}

"Primary" Angioplasty of the Right Internal Carotid Artery in a Patient with Contraindication for Thrombolytic Therapy

We report a case of a 63-year-old male patient with a previous history of ischemic stroke, motor sequelae and occlusion of the left internal carotid artery. He was admitted at the emergency room with an acute episode of a new ischemic stroke (4 hours after symptoms onset). The patient had an absolute contraindication for thrombolytic therapy due to a recent liver biopsy. Thus, we decided to perform an emergency angiography of the carotid and vertebral arteries. Culprit lesion was identified and we decided to implant a self-expandable stent, obtaining angiographic success and significant clinical improvement. Although we do not have strong evidence supporting the chosen strategy ("primary" angioplasty of the internal carotid artery), it seemed to be the best one to be used at the time.

DESCRIPTORS: Carotid arteries. Carotid artery diseases. Stroke. Angioplasty.

lutas à instituição desse tipo de tratamento, como realização de procedimentos invasivos nas duas semanas que antecedem sua infusão, acidente vascular encefálico isquêmico ou traumatismo cranioencefálico em período de três meses, e história pregressa de alguma hemorragia cerebral, entre outras. O objetivo do presente relato é descrever o manejo invasivo, com implante de stent carotídeo na fase aguda de acidente vascular encefálico isquêmico, em um indivíduo no qual o tratamento trombolítico era formalmente contraindicado, reportando sua evolução clínica intra-hospitalar.

\section{RELATO DO CASO}

Paciente do sexo masculino, 63 anos, com antecedentes de hipertensão arterial sistêmica, dislipidemia, diabetes tipo 2 não-dependente de insulina, tabagismo e hepatite $\mathrm{C}$. Além disso, o paciente era portador de marcapasso definitivo, implantado há três anos, em decorrência de bloqueio atrioventricular total. Apre- 
Attizzani GF, et al. Angioplastia "Primária" de Artéria Carótida Interna Direita em Paciente com Contraindicação à Terapia Trombolítica. Rev Bras Cardiol Invas. 2009;17(4):549-52.

sentou acidente vascular encefálico isquêmico há dois anos, permanecendo com sequela motora à direita. Havia realizado ecografia com Doppler de artérias carótidas naquela oportunidade, que evidenciou oclusão total da artéria carótida interna esquerda em sua origem, além de lesão moderada na origem da artéria carótida interna direita.

Familiares relataram que o paciente havia realizado biópsia hepática nove dias antes do atendimento atual. Além disso, fazia uso regular de sinvastatina $10 \mathrm{mg}$, uma vez por dia, ácido acetilsalicílico 100 mg, uma vez por dia (suspendeu para a biópsia e não reiniciou), enalapril $20 \mathrm{mg}$, duas vezes por dia, daonil $5 \mathrm{mg}$, duas vezes por dia, e hidroclorotiazida 12,5 mg, uma vez por dia. O motivo principal do atendimento foi o quadro súbito de "dificuldade de movimentação do lado esquerdo, sonolência e dificuldade de fala" (sic), iniciado há aproximadamente quatro horas.

Ao exame clínico, à parte a sequela prévia de diminuição (grau III) de força muscular em domínio D, encontrava-se em escala de Glasgow 12 (abertura ocular $=3$, resposta verbal $=3$, resposta motora $=6$ ), disartria e hemiparesia completa e desproporcionada à esquerda, de predomínio crural, força muscular grau IV em membro superior esquerdo e grau II no membro inferior esquerdo. O exame cardiovascular não apresentou alterações significativas, à exceção de pressão arterial de 170/100 mmHg. Tomografia de crânio sem contraste à admissão não exibia sinais de sangramentos intraparenquimatosos ou novas áreas de isquemia, e os exames laboratoriais apresentavam-se sem alterações dignas de nota (creatinina, 0,9 mg/dl; glicemia, $130 \mathrm{mg} / \mathrm{dl}$; e coagulograma, normal).

Paciente de alto risco em decorrência das comorbidades associadas, além de episódio prévio de acidente vascular encefálico com oclusão da artéria carótida interna esquerda e lesão moderada na origem da artéria carótida interna direita. Seria um candidato à realização de rt-PA intravenosa ou intra-arterial; entretanto, o fato de ter sido submetido a biópsia hepática recente contraindicava, de modo absoluto, tal tratamento. Assim, optou-se pela realização de angiografia de emergência das artérias carótidas e vertebrais.

\section{Angiografia}

A artéria femoral direita foi puncionada pela técnica de Seldinger modificada, sendo colocado um introdutor Medtronic 6 F. À angiografia de artérias vertebrais, não foram observadas lesões obstrutivas. A artéria carótida interna esquerda encontrava-se ocluída em sua origem (Figura 1) e a artéria carótida interna direita estava subocluída em seu terço proximal, com imagem negativa em seu interior sugestiva de trombo (Figuras 2 e 3), sendo indicada angioplastia da artéria carótida interna direita. O introdutor colocado inicialmente foi substituído por um Cordis $8 \mathrm{~F}$ (Cordis, Rio de Janeiro, Brasil) e a artéria carótida comum direita foi

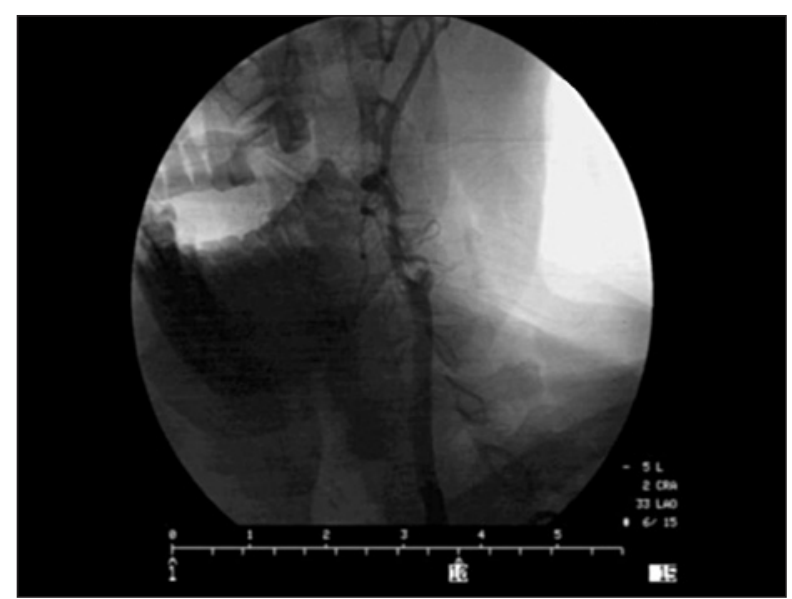

Figura 1 - Artéria carótida interna esquerda exibe obstrução total em sua origem.

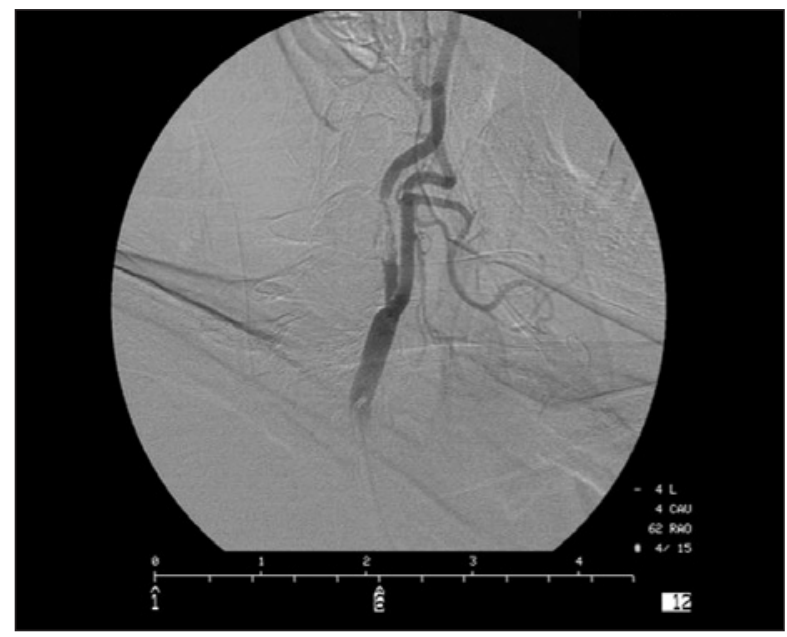

Figura 2 - Artéria carótida interna direita exibe suboclusão em seu terço proximal, com imagem negativa em seu interior sugestiva de trombo.

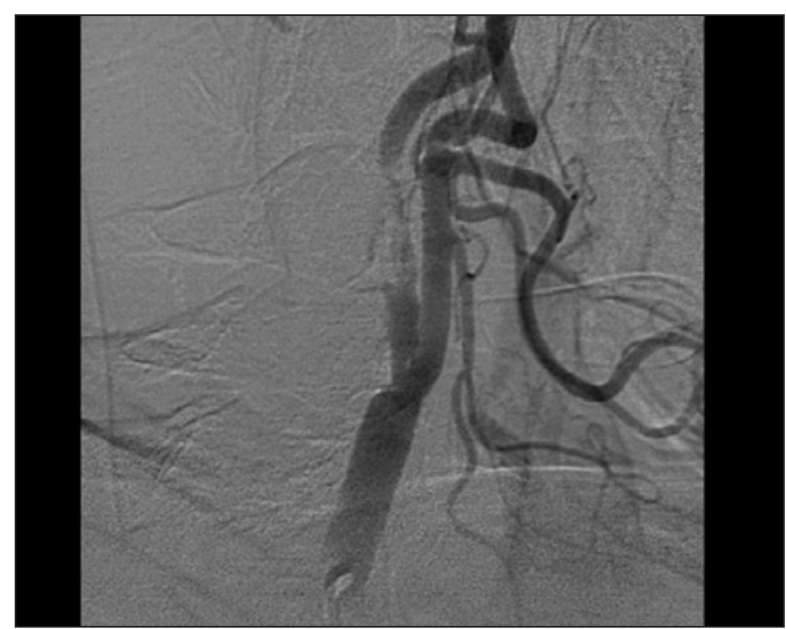

Figura 3 - Imagem da artéria carótida interna direita magnificada. 
Attizzani GF, et al. Angioplastia "Primária" de Artéria Carótida Interna Direita em Paciente com Contraindicação à Terapia Trombolítica. Rev Bras Cardiol Invas. 2009;17(4):549-52.

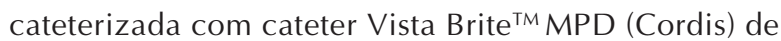
8 F. Angioguard RX ${ }^{\mathrm{TM}}$ (Cordis) foi posicionado distalmente à lesão da artéria carótida interna direita e, em seguida, o Stent Precise ${ }^{\mathrm{TM}}$ (Cordis) $6 \mathrm{~mm} \times 40 \mathrm{~mm}$ foi posicionado sob a lesão. O stent foi então liberado e realizada pós-dilatação intrastent com balão Amiia $^{\mathrm{TM}}$ (Cordis) $6 \mathrm{~mm} \times 20 \mathrm{~mm}$, com 10 atm. O dispositivo de proteção distal foi recolhido, obtendo-se, finalmente, resultado angiográfico satisfatório (Figura 4) e melhora substancial do fluxo sanguíneo cerebral (Figura 5).

\section{Evolução clínica}

O paciente apresentou boa evolução intra-hospitalar, com melhora do déficit motor (força muscular grau $\mathrm{V}$ em membro superior esquerdo e grau IV em membro inferior esquerdo) e normalização da fala. Recebeu alta hospitalar no oitavo dia, medicado com ácido acetilsalicílico $100 \mathrm{mg}$, uma vez por dia, clopidogrel $75 \mathrm{mg}$, uma vez por dia, enalapril $20 \mathrm{mg}$, duas vezes por dia, atorvastatina $40 \mathrm{mg}$, uma vez por dia, daonil $5 \mathrm{mg}$, três vezes por dia, metformina $500 \mathrm{mg}$, duas vezes por dia, hidroclorotiazida 12,5 mg, uma vez por dia, e amlodipina $5 \mathrm{mg}$, uma vez por dia.

\section{DISCUSSÃO}

O acidente vascular encefálico isquêmico deve ser encarado como emergência médica e demanda uma conduta pró-ativa por parte de toda a equipe envolvida no atendimento, incluindo médicos do serviço de emergência, neurologista e radiologista, bem como de toda a equipe de enfermagem e técnicos. Embora a terapia trombolítica intravenosa deva ser idealmente iniciada até três horas após o início dos sintomas, recentemente foi evidenciado benefício da ampliação da janela terapêutica do uso do rt-PA para intervalo de até 4,5 horas $^{6}$. Já o manejo invasivo desses indivíduos, com infusão intra-arterial de trombolítico, mostrou-se eficaz até seis horas após o início dos

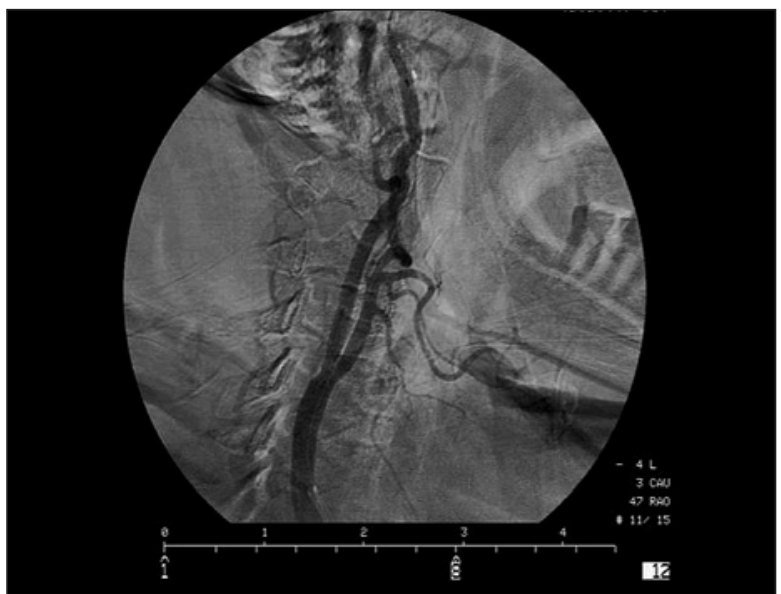

Figura 4 - Resultado após liberação do stent.
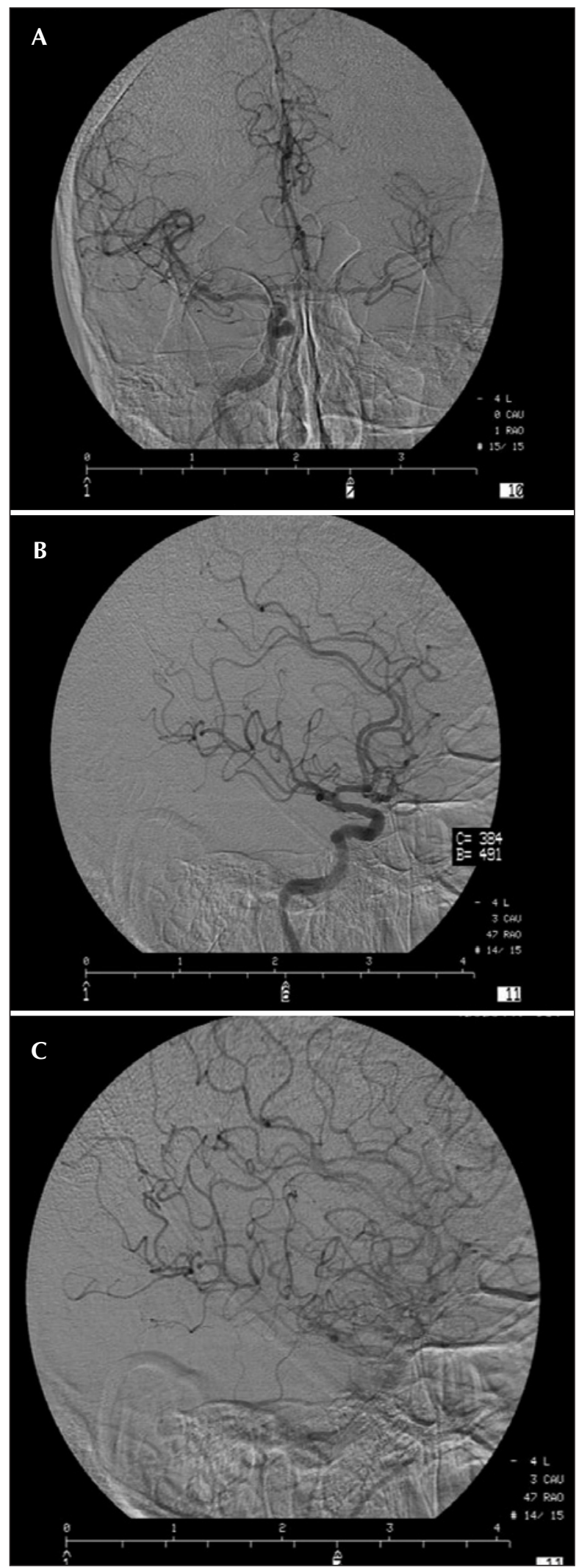

Figura 5 - Angiografia cerebral realizada com a técnica de subtração digital em projeção frontal (A) e oblíqua anterior direita, nas fases arterial (B) e arteriolar (C). 
sintomas ${ }^{7}$. Contudo, o uso dessa droga tem baixa probabilidade de lograr recanalização eficaz de oclusões das artérias carótidas internas ${ }^{8}$. A instituição de procedimento de revascularização na fase aguda, tanto por meio de endarterectomia como pelo implante de stents, carece de substratos robustos, constituindo-se sobretudo de pequenos registros e relatos de $\operatorname{casos}^{9-12}$. Jovin et al. ${ }^{13}$ estudaram 25 indivíduos submetidos a implante de stents na fase aguda de oclusão de artéria carótida interna, relatando taxas de sucesso que alcançaram $92 \%$ e baixos índices de complicações, atingindo melhora clínica significativa nas 24 horas após a intervenção, que se tornou ainda mais acentuada no seguimento de 30 dias. No presente relato, optamos por abordar o paciente invasivamente, após confirmarmos a ausência de sinais de hemorragia, dado seu elevado risco (acidente vascular encefálico prévio com oclusão da artéria carótida interna esquerda), relato prévio de lesão na artéria carótida interna direita e, sobretudo, em decorrência de contraindicação absoluta à infusão de trombolíticos. Alguns fatores que contribuíram para o êxito tanto angiográfico como clínico do procedimento realizado foram a apresentação relativamente precoce do paciente, associada à rápida tomada de decisão da equipe e à instituição do tratamento definitivo. Ressaltamos que o procedimento realizado neste caso necessita ser avaliado em grandes estudos randomizados, para que se possa estabelecer sua real segurança e eficácia, podendo, daí em diante, ser recomendado de modo rotineiro no tratamento da doença aqui descrita. Até que isso não ocorra, os tratamentos instituídos regularmente com esse fim deverão ser guiados pelas diretrizes vigentes.

\section{CONFLITO DE INTERESSES}

Os autores declararam inexistência de conflito de interesses relacionado a este artigo.

\section{REFERÊNCIAS BIBLIOGRÁFICAS}

1. Bonita R. Epidemiology of stroke. Lancet. 1992;339(8789): 342-4.
2. Adams HP Jr, Bendixen BH, Leira E, Chang $\mathrm{KC}$, Davis $\mathrm{PH}$, Woolson RF, et al. Antithrombotic treatment of ischemic stroke among patients with occlusion or severe stenosis of the internal carotid artery: a report of the Trial of Org 10172 in Acute Stroke Treatment (TOAST). Neurology. 1999;53(1):122-5.

3. Grubb RL Jr, Derdeyn CP, Fritsch SM, Carpenter DA, Yundt $\mathrm{KD}$, Videen TO, et al. Importance of hemodynamic factors in the prognosis of symptomatic carotid occlusion. JAMA. 1998;280(12):1055-60.

4. Derdeyn CP, Grubb RL Jr, Powers WJ. Cerebral hemodynamic impairment: methods of measurement and association with stroke risk. Neurology. 1999;53(2):251-9.

5. Sociedade Brasileira de Doenças Cerebrovasculares. Primeiro consenso brasileiro para trombólise no acidente vascular cerebral isquêmico agudo. Arq Neuropsiquiatr. 2002;60(3-A): 675-80.

6. Hacke W, Kaste M, Bluhmki E, Brozman M, Dávalos A, Guidetti $\mathrm{D}$, et al. Thrombolysis with alteplase 3 to 4.5 hours after acute ischemic stroke. N Engl J Med. 2008;359(13):1317-29.

7. Furlan A, Higashida R, Wechsler L, Gent M, Rowley H, Kase C, et al. Intra-arterial prourokinase for acute ischemic stroke. The PROACT II study: a randomized controlled trial. Prolyse in Acute Cerebral Thromboembolism. JAMA. 1999;282(21): 2003-11.

8. Kim YS, Garami Z, Mikulik R, Molina CA, Alexandrov AV, CLOTBUST Collaborators. Early recanalization rates and clinical outcomes in patients with tandem internal carotid artery/ middle cerebral artery occlusion and isolated middle cerebral artery occlusion. Stroke. 2005;36(4):869-71.

9. Meyer FB, Sundt TM Jr, Piepgras DG, Sandok BA, Forbes G. Emergency carotid endarterectomy for patients with acute carotid occlusion and profound neurological deficits. Ann Surg. 1986;203(1):82-9.

10. Spearman MP, Jungreis CA, Wechsler LR. Angioplasty of the occluded internal carotid artery. AJNR Am J Neuroradiol. 1995;16(9):1791-9.

11. Du Mesnil De Rochemont R, Sitzer M, Neumann-Haefelin T, Harmjanz A, Berkefeld J. Endovascular recanalization of acute atherothrombotic carotid artery occlusion holds up progressive stroke. Neuroradiology. 2004;46(7):583-6.

12. Kim SH, Qureshi Al, Levy El, Hanel RA, Siddiqui AM, Hopkins $\mathrm{LN}$. Emergency stent placement for symptomatic acute carotid artery occlusion after endarterectomy. Case report. J Neurosurg. 2004;101(1):151-3.

13. Jovin TG, Gupta R, Uchino K, Jungreis CA, Wechsler LR, Hammer MD, et al. Emergent stenting of extracranial internal carotid artery occlusion in acute stroke has a high revascularization rate. Stroke. 2005;36(11):2426-30. 